EXTENDED REPORT

\title{
Treatment continuation in patients receiving biological agents or conventional DMARD therapy
}

\section{A Zink, J Listing, S Kary, P Ramlau, M Stoyanova-Scholz, K Babinsky, U von Hinueber, E Gromnica-lhle, S Wassenberg, C Antoni, P Herzer, J Kekow, M Schneider, R Rau}

See end of article for authors' affiliations

.....................

Correspondence to Dr Prof Angela Zink, Deutsches RheumaForschungszentrum Berlin Forschungsbereich Epidemiologie,

Schumannstr $21 / 22, \mathrm{D}$

10117 Berlin, Germany;

Zink@DRFZ.de

Accepted 7 February 2005

Published Online first

11 February 2004
Objective: To compare drug continuation rates in patients with rheumatoid arthritis who start on a biological agent and in a control group of patients with a change in disease modifying antirheumatic drug (DMARD) treatment after previous DMARD failure.

Methods: Patients with rheumatoid arthritis enrolled in the German biologics register between May 2001 and September 2003 were included in the study. Data were available for 511 patients treated with etanercept, 343 with infliximab, 70 with anakinra, and 599 controls. Propensity scores were used to select a subsample of patients from the control group who were likely to be treated with biological agents because of their disease severity, as well as comparable infliximab and etanercept cases.

Results: Treatment continuation after 12 months was similar for etanercept $168.6 \% 195 \%$ confidence interval, $62 \%$ to $75 \%))$ and infliximab $(65.4 \%(58 \%$ to $73 \%))$ but lower for anakinra $(59 \%(41 \%$ to $77 \%))$. Treatment continuation was more likely for patients on combinations of biological agents and DMARDs than for those on infliximab or etanercept alone. Patients treated with biological agents were more severely ill than those in the control group and had more previous DMARD failures. After adjustment for baseline differences, the continuation rates were higher in patients treated with biological agents than in comparable control patients treated with leflunomide or leflunomide/methotrexate.

Conclusions: Treatment continuation of biological agents in clinical practice is less likely than in randomised clinical trials but more likely than in comparable controls treated with conventional DMARDs.
C ytokine inhibitors have opened up new and promising treatment options in rheumatoid arthritis. Randomised clinical trials have demonstrated the efficacy of cytokine inhibitors in reducing inflammation as well as in inhibiting joint destruction in active rheumatoid arthritis, particularly in patients not responding to conventional disease modifying antirheumatic drugs (DMARDs). ${ }^{1-5}$

Although trial based evidence and clinical experience to date are encouraging, there is still insufficient information on the long term safety and continuing efficacy of biological agents in the general population with rheumatoid arthritis and in high risk patient groups (for example, patients with serious comorbidity). Concerns have been raised over the risk of reactivation of tuberculosis, an increase in other severe infections, and rare but serious events such as drug induced lupus or demyelinating disease, as well as the unknown risk of malignancies.

Long term observational studies on patients treated with biological agents have various specific strengths. They represent the full spectrum of patients undergoing treatment, including those who would be excluded from clinical trials owing to severe comorbidity. They allow comparison of different treatments and treatment regimens (including offlabel use) within a single study, and-because of their long term approach-they present the outcomes of exposure to multiple drugs (given concomitantly or subsequently). Additionally, they provide information on the cost-effectiveness of new treatments in real life clinical practice.

Various European countries have established long term registers of patients treated with biological agents. The German register has enrolled an inherent control group which consists of patients with a change of conventional DMARD therapy because of inadequate disease control or poor tolerability of at least one previous DMARD. These patients have been followed up with the same protocol as the patients treated with biological agents. This will allow us to compare the results in the biologics groups with the overall risks of the disease and its present treatment.

In this paper we address the following questions. First, what is the baseline clinical status of patients starting on a biological treatment for the first time in Germany, compared with a control group of patients with at least one failure on conventional DMARD therapy? Second, what is the drug continuation rate over the first 12 months in patients receiving the various biological agents and in the subsamples of the control group?

We report data from the German biologics register for patients enrolled up to I September 2003. At that point, data were available in the biological agents group for patients initially treated with etanercept, infliximab, or anakinra. Patients in the adalimumab group are not included in this analysis owing to the later start of enrolment.

\section{METHODS}

\section{Background}

In 2001, the German Society of Rheumatology issued a guideline on the prescription of biological treatments, ${ }^{6}$ recommending that patients who have failed to respond to or have not tolerated at least two DMARDs, including methotrexate, should be treated with cytokine inhibitors. In conjunction with this recommendation, the German Society of Rheumatology invited all rheumatologists to contribute to

Abbreviations: DAS28, 28 joint disease activity score; DMARD, disease modifying antirheumatic drug; $\mathrm{FFbH}$, Hannover functional status questionnaire; SJC, swollen joint count; TJC, tender joint count 


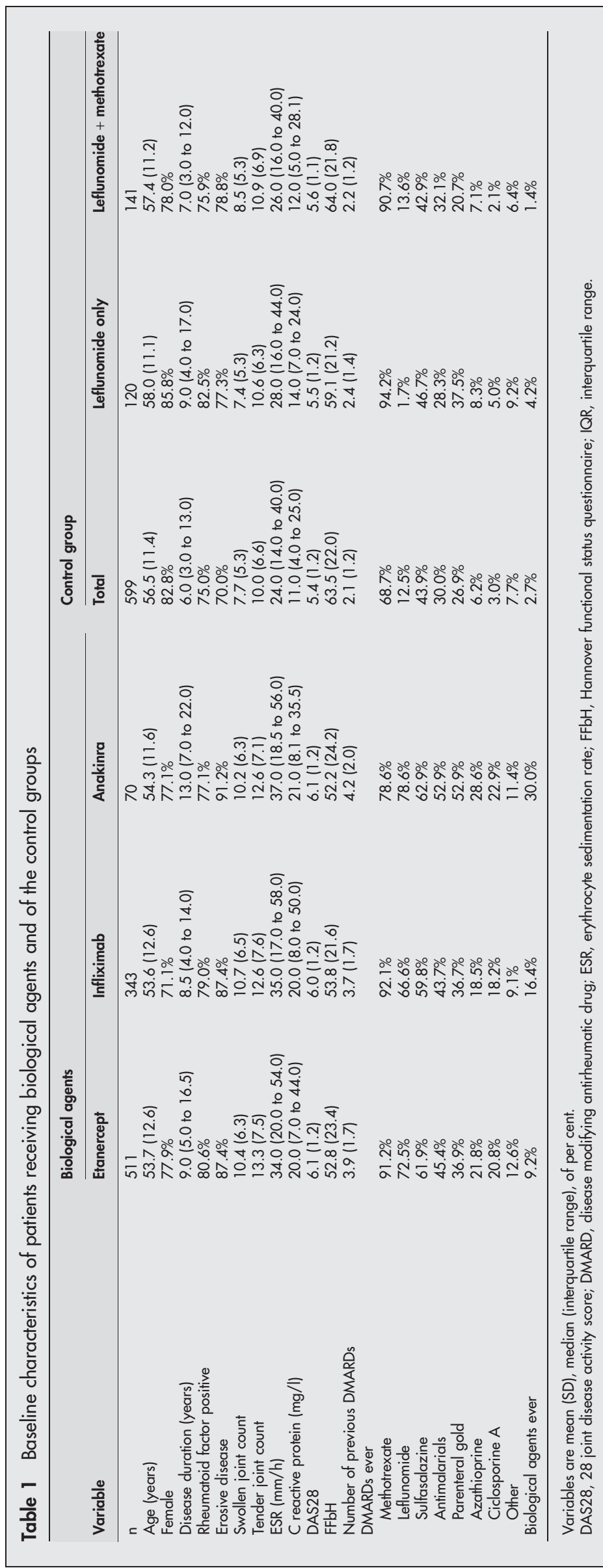

a national register. The epidemiology unit at the German Rheumatism Research Centre was charged with maintaining the register, and an advisory board was established by the German Society of Rheumatology. The objectives of this prospective cohort study (known as RABBIT, which is the German acronym for: rheumatoid arthritis-observation of biologic therapy) are to describe the long term effectiveness of treatment with biological agents with regard to treatment continuation and clinical outcomes, to study the long term hazards of treatment with cytokine inhibitors, and to establish the direct and indirect costs of biological versus conventional DMARD treatment.

\section{Patients}

Patients aged 18 to 75 years meeting the American College of Rheumatology (ACR) criteria for rheumatoid arthritis were eligible as "cases" if a new treatment with infliximab, etanercept, or anakinra (since January 2003) was started, and as "controls" if a conventional DMARD treatment was begun after failure of at least one previous therapy. Patients could also be enrolled in the control group if another DMARD was added to an existing regimen. Patients were required to give written informed consent at the time of enrolment. The study protocol was approved by the ethics committee of the Charité Hospital, University of Berlin and, where necessary, by the local ethics committee of the participating rheumatology unit.

Patient recruitment is ongoing. In the following analyses patients enrolled up to I September 2003 were included. All follow up data available to March 2004 were used.

\section{Procedures}

Each rheumatologist agreeing to participate was provided with the study protocol, study information, and informed consent documents for the patients, as well as case report forms. The study protocol stipulates that treatment decisions were not to be influenced by the principal investigators, the scientific advisory board, or the pharmaceutical companies sponsoring the register. For reasons of full transparency, all participating rheumatologists received a copy of the contract between the German Rheumatism Research Centre and the four pharmaceutical companies. The contract specifies that full responsibility for the conduct of the study, data ownership, and publication rights are in the hands of the principal investigators.

\section{Assessments}

At each visit, the treating rheumatologist recorded a 28 joint count of tender (TJC) and swollen (SJC) joints, erythrocyte sedimentation rate (ESR; Westergren method), C reactive protein, morning stiffness, DMARD or biological treatment including details of start/end, reasons for treatment termination, concomitant treatment with glucocorticoids and non-steroidal anti-inflammatory drugs (NSAIDs), and adverse events. In addition, patients assessed their pain, general health, or fatigue on numerical rating scales from 0 to 10 and reported sociodemographic details. The Hannover functional status questionnaire (Funktionsfragebogen Hannover, FFbH) was to be completed every six months. This instrument is comparable to the health 
Table 2 Current prescription of DMARDs in patients receiving biologics and in the control groups

\begin{tabular}{llllc}
\hline DMARDs currently (\%) & Etanercept & Infliximab & Anakinra & Control \\
\hline No DMARD & 49.7 & 10.5 & 30.0 & NA \\
Methotrexate alone & 32.7 & 63.8 & 60.0 & 20.2 \\
Leflunomide alone & 7.8 & 13.4 & 5.7 & 20.0 \\
Other single therapy & 3.3 & 1.7 & 1.4 & 4.7 \\
Combination methotrexate + leflunomide & 1.8 & 3.8 & 1.4 & 23.5 \\
Other combination of 2 DMARDs & 3.5 & 4.7 & 1.4 & 24.0 \\
Combination of 3 DMARDs & 1.2 & 2.0 & 0.0 & 7.5 \\
\hline
\end{tabular}

DMARD, disease modifying antirheumatic drug; NA, not assessed.

assessment questionnaire (HAQ), and scores can be transformed from one questionnaire to the other. ${ }^{7}$ The disease activity score based on 28 joint counts (DAS28) was calculated. ${ }^{8}$ The case report forms were sent by fax to the study centre. Queries were sent back in the case of incomplete or inconsistent data. The minimum valid data asked for in every case included the baseline characteristics, and at follow up the start and end of DMARD or biological treatment, reasons for treatment termination, and detailed descriptions of adverse events.

\section{Statistics}

The $\chi^{2}$ test and the non-parametric Kruskal-Wallis test were used to compare the baseline characteristics of the patients. The Kaplan-Meier method was applied to calculate the probability of treatment continuation. Three of these survival analyses were carried out for each drug. The first analysis considered treatment termination overall, resulting from adverse events, lack of efficacy, or miscellaneous causes such as non-compliance. In the second analysis, only those treatment terminations were considered where adverse events were cited as at least one reason for stopping. In the third analysis, the same procedure was followed for lack of efficacy. Treatment terminations resulting from partial remission were calculated as censored data. Only the continuation of the new treatment applied at study entry was investigated; subsequent treatment episodes were not considered. Different Kaplan-Meier curves were compared using the log-rank test. The corresponding 95\% confidence intervals (CI) of the continuation rates were estimated by the Hall-Wellner method. Cox proportional hazard models were used to investigate the effect of possible risk factors on treatment termination (number of previous DMARDs, rheumatoid factor, DAS28, SJC, TJC, FFbH, disease duration, age, and sex).

As patients in the control group and those in the biological agents groups differed significantly in their baseline characteristics, propensity scores were calculated in order to select a more comparable subgroup of patients from the control

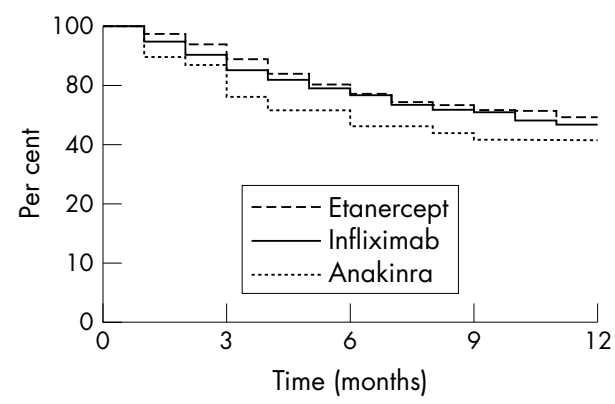

Figure 1 Probability of treatment continuation in patients receiving biological agents. group. Multivariate logistic regression was applied to estimate the likelihood (propensity score) of being treated with tumour necrosis factor (TNF) inhibitors (infliximab or etanercept). The following baseline characteristics were included in the multivariate logistic regression analysis: age, number of previous DMARDs, DAS28, and FFbH. Each of these variables discriminated significantly between cases and controls. Twenty seven of 120 patients treated at study entry with leflunomide alone and 21 of 141 patients treated with leflunomide and methotrexate but only six of 121 patients treated with methotrexate alone fulfilled the criterion of a propensity score $>50 \%$ of being treated with TNF inhibitors. We therefore used the 48 patients from the leflunomide subgroups as fulfilling the above mentioned criterion for the comparisons.

\section{RESULTS}

Between 1 May 2001 and 1 September 2003, 1523 patients from 109 centres were entered into the RABBIT database. In this population, 599 patients had a change in their conventional DMARD therapy (control group), and 511 patients started treatment with etanercept, 343 with infliximab, and 70 with anakinra.

\section{Patient characteristics}

Table 1 shows the baseline clinical status of patients treated with the individual biological drugs compared with patients in the control group. Two large subgroups of the control group (leflunomide alone or leflunomide plus methotrexate) are shown separately. In patients treated with biological agents, the mean age in all groups was 54 years and mean disease duration ranged from 9 to 13 years. The patients had very active disease, with a mean of more than 10 swollen joints and raised ESR and C reactive protein. DAS28 and functional status were similar in the biologics groups. Patients in all three groups had a long treatment history with DMARDs. The vast majority had been treated with methotrexate before, and about three quarters with leflunomide. Other DMARDs such as sulfasalazine or antimalarials had been tried in a large number of patients, leading to an average number of previous DMARDs of 3.9 to 4.2 .

Patients in the control group had significantly shorter disease duration $(\mathrm{p}<0.001)$, a lower prevalence of erosive disease, and a lower mean DAS28 (5.4 $v 6.0$ and 6.1, $\mathrm{p}<0.001$ ) than those in the biological agents groups. Patients treated with leflunomide or leflunomide/methotrexate had a greater number of previous DMARD failures $(p<0.001)$, a higher DAS28 $(p=0.002)$, and a higher prevalence of erosive disease $(p=0.001)$ than the other patients in the control group. Thus they were more suitable than the rest of the control group in terms of comparability with the patients in the biological agents groups.

The major difference between the biological agents group and the control group was the number of previous DMARD failures: patients receiving biological agents had almost twice 


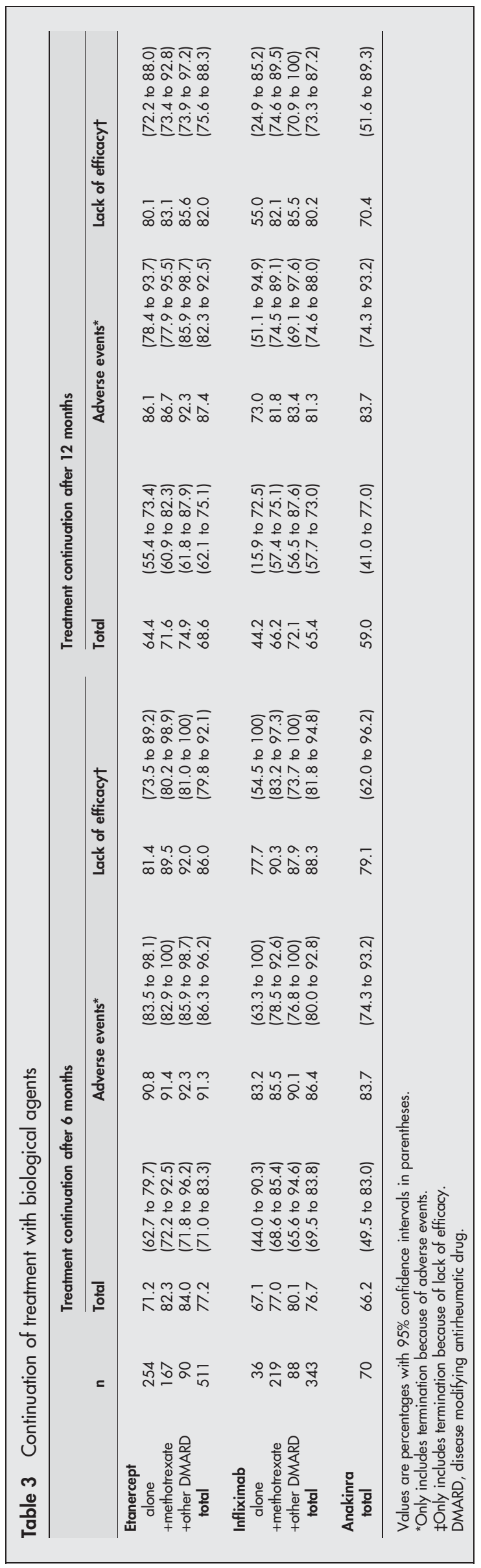

as many DMARD failures on average. Most had previous experience with methotrexate plus a variety of other DMARDs. In addition, only $1.4-4.2 \%$ of the patients in the control group had been treated with a biological agent previously, compared with $30 \%$ of those receiving anakinra, $16.4 \%$ of those receiving infliximab, and $9.2 \%$ of those receiving etanercept.

\section{DMARD therapy in combination with a biological agent}

Although infliximab is approved for use in combination with methotrexate, $10.5 \%$ of the patients were treated with infliximab alone at study entry and approximately one quarter of the patients were treated with infliximab in combination with another DMARD (table 2). More than half the etanercept patients were treated concomitantly with conventional DMARDs. In $6.7 \%$ of the infliximab patients and $4.7 \%$ of the etanercept patients, two or three DMARDs were prescribed in addition to the biological agent.

\section{Treatment continuation}

Similar drug continuation rates were found for etanercept $(69 \%(95 \%$ CI, $62 \%$ to $75 \%))$ and infliximab (65\% (58\% to $73 \%)$ ) for the first 12 months of observation (fig 1). The continuation rates for anakinra (59\% (41\% to $77 \%)$ ) were significantly lower $(\mathrm{p}=0.004$ anakinra $v$ etanercept; $\mathrm{p}=0.03$ anakinra $v$ infliximab).

Predictors of premature treatment termination were the number of previous DMARDs (hazard ratio (HR) $=1.09(95 \%$ CI, 1.01 to 1.18$)$ ), rheumatoid factor $(\mathrm{HR}=1.53$ ( 1.09 to $2.16))$, and greater age $(\mathrm{HR}=1.01(1.00$ to 1.02$))$ but not DAS28, TJC, or SJC at baseline.

The drug continuation rates for etanercept in combination with methotrexate or another DMARD tended to be higher $(p=0.11$ and $p=0.07)$ than for etanercept alone (table 3$)$. In the infliximab group this difference between single and combination therapy was even more apparent. Following adjustment for risk factors of premature treatment termination mentioned above, the hazard ratio of treatment termination for infliximab alone in comparison to infliximab/methotrexate was 1.9 (95\% CI, 1.1 to 3.1$)$. For etanercept alone versus etanercept/DMARD the HR was 1.3 (0.9 to 1.8$)$.

Reasons for treatment termination were specified by the rheumatologist in nearly all cases $(288 / 290)$ where treatment with a biological agent was discontinued. In 28 cases (12 with infliximab, 15 with etanercept, and one with anakinra,

Table 4 Subgroups of patients with propensity score $>50 \%$ for treatment with biologics (see Methods)

\begin{tabular}{lll}
\hline Variable & $\begin{array}{l}\text { Etanercept or } \\
\text { infliximab }\end{array}$ & Leflunomide \\
\hline $\mathrm{n}$ & 563 & 48 \\
Age (years) & $52.7(12.5)$ & $53.2(11.1)$ \\
Female & $78.2 \%$ & $87.5 \%$ \\
Disease duration (years) & $10(6$ to 17$)$ & $12(6$ to 19$)$ \\
Rheumatoid factor positive & $83.3 \%$ & $81.3 \%$ \\
Erosive disease & $89.3 \%$ & $85.4 \%$ \\
Swollen joint count & $11.6(6.3)$ & $10.9(5.6)$ \\
Tender joint count & $14.4(7.3)$ & $14.4(6.1)$ \\
ESR (mm/h) & $38(22$ to 58$)$ & $30(20$ to 50$)$ \\
C reactive protein (mg/l) & $23(8$ to 52$)$ & $16(9$ to 42$)$ \\
DAS28 & $6.4(1.1)$ & $6.4(0.9)$ \\
FFbH & $49.1(22.6)$ & $50.4(21.4)$ \\
Number of previous DMARDs & $4.6(1.5)$ & $3.9(1.2)$ \\
\hline
\end{tabular}

Values are mean (SD) or median (interquartile range) unless specified. DAS28, 28 joint disease activity score; DMARD, disease modifying antirheumatic drug; ESR, erythrocyte sedimentation rate; $F F b H$, Hannover functional status questionnaire. 
total $9.8 \%$ ) more than one reason was reported (lack of efficacy, adverse event, non-compliance).

Table 3 shows the drug continuation rates for the three biological agents alone or in combination with DMARDs after six and 12 months, taking specific reasons for discontinuation into account. When only terminations because of adverse events were considered, the continuation rates after 12 months ranged from $81.3 \%$ for infliximab (total) to $87.4 \% \%$ for etanercept (total). The rates of treatment terminations because of lack of efficacy were very similar for infliximab and etanercept in combination with methotrexate or other DMARD but lower for anakinra and infliximab alone (table 3). The probability of discontinuation for other reasons was very low. Furthermore, in two cases receiving etanercept, seven receiving infliximab, and one receiving anakinra, treatment was stopped because of partial remission. In eight of these 10 cases, treatment with methotrexate or leflunomide was continued.

Controls treated with leflunomide or leflunomide/ methotrexate did not differ significantly in their treatment continuation rates: leflunomide $76.5 \%$ and leflunomide/methotrexate $72.7 \%$ after six months; leflunomide $67.8 \%$ and leflunomide/methotrexate $62.4 \%$ after 12 months $(p=0.28)$. In both groups the major reasons for treatment termination were adverse events (27.6\%) and lack of efficacy (13.2\%).

However, these patients were not fully comparable with the patients treated with biological agents. We therefore generated propensity scores that predicted the start of treatment with a biological agent (see Methods). This procedure identified 48 controls receiving leflunomide or leflunomide/methotrexate who had a high likelihood of treatment with a biological agent, based on their clinical status at study entry. These patients had baseline characteristics similar to 563 patients treated with TNF inhibitors (etanercept $\mathrm{n}=350$, infliximab $\mathrm{n}=213$ ) who also met the criterion of a propensity score $>50 \%$ (table 4 ). The two groups differed significantly only in terms of the numbers of previous DMARDs ( 4.6 v 3.9).

Nevertheless, the patients in the leflunomide subgroup had lower treatment continuation rates $(64.1 \%$ after six months, $51.0 \%$ after 12 months) than patients receiving TNF inhibitors (fig 2, $\mathrm{p}=0.058$ ). This result was confirmed in a Cox regression analysis, taking risk factors for premature treatment termination into account. Following adjustment for age, rheumatoid factor, and number of previous DMARDs, there was a significantly increased hazard ratio of $1.7(p=0.025)$ for treatment termination with leflunomide in comparison with infliximab/etanercept.

\section{DISCUSSION}

Even though rheumatologists today have access to far more effective drugs than they did a decade ago, a cure remains

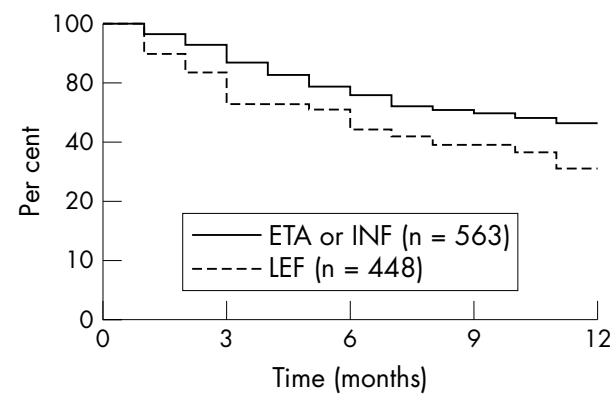

Figure 2 Probability of treatment continuation in patients with an increased likelihood of being treated with tumour necrosis factor (propensity score $>50 \%$ ). elusive and all available agents have to be given continuously in order to suppress inflammation and joint destruction. It is known that a majority of patients respond well to methotrexate therapy, which has high drug continuation rates.' However, if methotrexate alone is insufficient or not tolerated, other therapeutic options have to be chosen. Much less is known about the long term continuation of the new drugs which are given in the risk group of patients with methotrexate failure.

The aim of this study was to compare treatment continuation rates of patients with rheumatoid arthritis treated in daily rheumatological practice with one of the cytokine inhibitors etanercept, infliximab, or anakinra or with leflunomide or a combination of methotrexate and leflunomide over the first 12 months. In order to select controls as similar as possible to the patients treated with biological agents, attention was focused exclusively on those patients in the control group who needed more effective therapy than methotrexate alone or who had intolerance to methotrexate.

Not unexpectedly, drug continuation over one year in our observational data was lower than in the major randomised clinical trials. In the ATTRACT trial ${ }^{2}$ drug continuation of infliximab plus methotrexate was $73 \%$. The TEMPO trial $^{10}$ showed drug continuation of $76 \%$ for etanercept alone and $84 \%$ for etanercept plus methotrexate. For anakinra, drug continuation over 24 weeks was $78 \%$ in the $1 \mathrm{mg} / \mathrm{kg} / \mathrm{day}$ group in the study by Cohen et al. ${ }^{11}$ Leflunomide alone had a drug continuation rate of $80 \%$ over 24 weeks in the study by Dougados et $a l^{12}$ and $77 \%$ in the study by Kremer et al..$^{13}$ One possible explanation for the higher treatment continuation in clinical trials relates to the nature of the trials themselves and the wish of both the investigator and the patient to persist, whereas in normal clinical practice they may decide to stop the drug or to change over.

The most important risk factor for treatment termination in our data was a large number of previous treatment failures. Two or more treatment failures have not been used as an inclusion criterion in clinical trials but are a cue for starting biological therapy in clinical practice. Thus the differences compared with clinical trials are not surprising because our data reflect routine day to day care in contrast to randomised trials with their rigorous protocols and restricted patient inclusion criteria. Reports from everyday rheumatology practice reveal data that resemble our own findings far more closely than those from randomised trials. In the Netherlands, Flendrie et al found one year drug continuation rates of $66 \%$ for infliximab and $74 \%$ for etanercept ${ }^{14}$ in patients treated with a biological agent for the first time; these findings are very similar to our data for infliximab $(65 \%)$ and higher than those for etanercept $(69 \%$ in our study). Chung et al reported a one year continuation rate for infliximab of $71 \%$ from an inception cohort in Canada, ${ }^{15}$ and this compares well with our data. The higher efficacy of etanercept in combination with methotrexate than of etanercept alone reported by van Vollenhoven et $a l^{16}$ from the Stockholm $\mathrm{TNF} \alpha$ registry is in agreement with our finding of higher continuation rates in etanercept combination therapy.

It has to be born in mind that treatment cessation is subject to within-physician variation. This is especially true for the reasons given in patients who experienced a relative lack of efficacy as well as an adverse drug reaction. Thus the total treatment continuation rates are more important than the rates for the various reasons. Furthermore, it is relevant that the physicians enrolled patients for all groups simultaneously. This allows comparisons between patients treated with infliximab or etanercept and those receiving conventional DMARD if the differences in the patient characteristics are taken into account. 
Geborek $e t$ al $^{17}$ found very low drug continuation rates for leflunomide of about $40 \%$ after 12 months in a comparable register of patients with previous DMARD failures; our data indicated relatively better performance for leflunomide. However, after controlling for disease severity and other factors influencing drug continuation, the TNF inhibitors in our data had higher continuation rates than leflunomide. The comparison of treatment continuation in patients treated with leflunomide or leflunomide/methotrexate on the one hand and TNF inhibitors on the other using the propensity score method showed that adjustment for background risk is essential in order to put treatment results into context. However, our results need further confirmation as they are based only on a rather small number of control patients.

\section{Conclusions}

This study is the first to show data on treatment continuation with various combinations of biological agents and conventional DMARDs. The data suggest that combination of both etanercept and infliximab with DMARDs such as methotrexate or leflunomide leads to higher treatment continuation. However, the data on combination with leflunomide or other DMARDs require confirmation by longer observation in the register and by other observational studies.

\section{ACKNOWLEDGEMENTS}

RABBIT is supported by an unconditional, joint grant from Essex Pharma (since 2001), Wyeth Pharma (since 2001), Amgen (since 1/ 2003), and Abbott (since 9/2003).

We wish to thank those rheumatologists who enrolled large numbers of cases (30 or more each): A Kapelle, Hoyerswerda; W Demary, Hildesheim; G-R Burmester, Berlin; K Rockwitz, Goslar; E Edelmann, Bad Aibling; E Wilden, Cologne; T Karger, Cologne; H-E Schroeder, Dresden; T Klopsch, Neubrandenburg; R Dockhorn, Weener; A Graessler, Pirna; K Krueger, Munich; W Liman, Hagen; H Soerensen, Berlin; WL Gross, Lübeck/Bad Bramstedt. We thank U Kamenz and K Peters for their careful monitoring of the study.

\section{Authors' affiliations}

A Zink, J Listing, S Kary, P Ramlau, German Rheumatism Research Centre, Berlin, Germany

M Stoyanova-Scholz, Department of Rheumatology, Klinikum Duisburg, Essen, Germany

K Babinsky, Practising rheumatologist, Halle, Germany

U von Hinueber, Practising rheumatologist, Hildesheim, Germany

E Gromnica-Ihle, Rheumaklinik Berlin-Buch, Berlin, Germany

S Wassenberg, Evangelisches Fachkrankenhaus, Ratingen, Germany

C Antoni, Institute for Clinical Immunology and Rheumatology, University of Erlangen, Erlangen, Germany

P Herzer, Practising rheumatologist, Munich, Germany

J Kekow, Clinic for Rheumatology, University of Magdeburg,

Magdeburg, Germany

M Schneider, Department of Rheumatology, Heinrich Heine University, Duesseldorf, Germany

R Rau, Ev Fachkrankenhaus, Ratingen, Germany

\section{REFERENCES}

1 Maini R, St Clair EW, Breedveld F, Furst D, Kalden J, Weisman M, et al. Infliximab (chimeric anti-tumour necrosis factor alpha monoclonal antibody) versus placebo in rheumatoid arthritis patients receiving concomitant methotrexate: a randomised phase III trial. ATTRACT Study Group. Lancet 1999:354:1932-9.

2 Lipsky PE, van der Heijde DM, St Clair EW, Furst DE, Breedveld FC, Kalden JR, et al. Infliximab and methotrexate in the treatment of rheumatoid arthritis. Anti-Tumor Necrosis Factor Trial in Rheumatoid Arthritis with Concomitant Therapy Study Group. N Engl J Med 2000;343:1594-602.

3 Moreland LW, Schiff MH, Baumgartner SW, Tindall EA, Fleischmann RM Bulpitt KJ, et al. Etanercept therapy in rheumatoid arthritis. A randomized, controlled trial. Ann Intern Med 1999;130:478-86.

4 Genovese MC, Bathon JM, Martin RW, Fleischmann RM, Tesser JR, Schiff MH, et al. Etanercept versus methotrexate in patients with early rheumatoid arthritis: two-year radiographic and clinical outcomes. Arthritis Rheum 2002;46: 1443-50.

5 Cohen S, Hurd E, Cush J, Schiff M, Weinblatt ME, Moreland LW, et al. Treatment of rheumatoid arthritis with anakinra, a recombinant human interleukin-1 receptor antagonist, in combination with methotrexate: results of a twenty-four-week, multicenter, randomized, double-blind, placebocontrolled trial. Arthritis Rheum 2002;46:614-24.

6 [Recommendations of the German Society of Rheumatology for therapy with tumor necrosis factor inhibitors. Pharmacotherapy Committee of the German Society of Rheumatology]. Z Rheumatol 2000;59:291-2.

7 Lautenschlaeger J, Mau W, Kohlmann T, Raspe HH, Struve F, Bruckle W, et al. Vergleichende Evaluation einer deutschen Version des Health Assessment Questionnaires (HAQ) und des Funktionsfragebogens Hannover (FFbH). [Comparative evaluation of a German version of the Health Assessment Questionnaire (HAQ) and the Hanover Functional Status Questionnaire (HFSQ)]. Z Rheumatol 1997;56:144-55

8 Prevoo ML, van't Hof MA, Kuper HH, van Leeuwen MA, van de Putte LB, van Riel PL. Modified disease activity scores that include twenty-eight-joint counts. Development and validation in a prospective longitudinal study of patients with rheumatoid arthritis. Arthritis Rheum 1995;38:44-8.

9 Hoekstra M, van de Laar MA, Bernelot Moens HJ, Kruijsen MW, Haagsma CJ. Longterm observational study of methotrexate use in a Dutch cohort of 1022 patients with rheumatoid arthritis. J Rheumatol 2003;30:2325-9.

10 Klareskog L, van der HD, de Jager JP, Gough A, Kalden J, Malaise M, et al. Therapeutic effect of the combination of etanercept and methotrexate compared with each treatment alone in patients with rheumatoid arthritis: double-blind randomised controlled trial. Lancet 2004;363:675-81.

11 Cohen S, Hurd E, Cush J, Schiff M, Weinblatt ME, Moreland LW, et al. Treatment of rheumatoid arthritis with anakinra, a recombinant human interleukin-1 receptor antagonist, in combination with methotrexate: results of a twenty-four-week, multicenter, randomized, double-blind, placebocontrolled trial. Arthritis Rheum 2002;46:614-24.

12 Dougados M, Emery P, Lemmel EM, de la SR, Zerbini CA, Brin S, et al. Efficacy and safety of leflunomide and predisposing factors for treatment response in patients with active rheumatoid arthritis: RELIEF 6-month data. $J$ Rheumatol 2003;30:2572-9.

13 Kremer JM, Genovese MC, Cannon GW, Caldwell JR, Cush JJ, Furst DE, et al. Concomitant leflunomide therapy in patients with active rheumatoid arthritis despite stable doses of methotrexate. A randomized, double-blind, placebocontrolled trial. Ann Intern Med 2002;137:726-33.

14 Flendrie M, Creemers MC, Welsing PM, den Broeder AA, van Riel PL. Survival during treatment with tumour necrosis factor blocking agents in rheumatoid arthritis. Ann Rheum Dis 2003;62(suppl 2):ii30-ii33.

15 Chung C, Mallon C, Spady B, Russell AS, Maksymovych WP. Survival analysis of treatment with leflunomide, combination leflunomide/methotrexate, and infliximab for rheumatoid arthritis in routine clinical practice. Arthritis Rheum 2003;48(suppl 9):S334.

16 van Vollenhoven RF, Ernestam S, Harju A, Bratt J, Klareskog L. Etanercept versus etanercept plus methotrexate: a registry-based study suggesting that the combination is clinically more efficacious. Arthritis Res Ther 2003;5:R347-51

17 Geborek P, Crnkic M, Petersson IF, Saxne T. Etanercept, infliximab, and leflunomide in established rheumatoid arthritis: clinical experience using a structured follow up programme in southern Sweden. Ann Rheum Dis 2002;61:793-8. 